University of Wollongong

Research Online

Faculty of Engineering - Papers (Archive)

Faculty of Engineering and Information

Sciences

2003

\title{
Mechanisms of limitation and nature of field dependence of critical current in HTS epitaxial YBaCuO films
}

V. M. Pan

Institute for Metal Physics, Ukraine

E. A. Pashitskii

Institute of Physics, Ukraine

S. M. Ryabchenko

Institute of Physics, Ukraine

V. A. Komashko

Institute for Metal Physics, Ukraine

A. V. Pan

University of Wollongong, pan@uow.edu.au

See next page for additional authors

Follow this and additional works at: https://ro.uow.edu.au/engpapers

Part of the Engineering Commons

https://ro.uow.edu.au/engpapers/57

\section{Recommended Citation}

Pan, V. M.; Pashitskii, E. A.; Ryabchenko, S. M.; Komashko, V. A.; Pan, A. V.; Dou, S. X.; Semenov, A. V.; Tretiatchenko, C. G.; and Fedotov, Y. V.: Mechanisms of limitation and nature of field dependence of critical current in HTS epitaxial YBaCuO films 2003.

https://ro.uow.edu.au/engpapers/57

Research Online is the open access institutional repository for the University of Wollongong. For further information contact the UOW Library: research-pubs@uow.edu.au 


\section{Authors}

V. M. Pan, E. A. Pashitskii, S. M. Ryabchenko, V. A. Komashko, A. V. Pan, S. X. Dou, A. V. Semenov, C. G. Tretiatchenko, and Y. V. Fedotov 


\title{
Mechanisms of Limitation and Nature of Field Dependence of Critical Current in HTS Epitaxial YBaCuO Films
}

\author{
Vladimir M. Pan, Ernst A. Pashitskii, Sergey M. Ryabchenko, Valentin A. Komashko, Alexey V. Pan, S. X. Dou, \\ Alexey V. Semenov, Constantin G. Tretiatchenko, and Yuri V. Fedotov
}

\begin{abstract}
Magnetic field and temperature dependencies of the critical current density, $J_{c}(H \| c, T)$ were measured by SQUIDmagnetometry, ac magnetic susceptibility, and dc transport current techniques in the single-crystalline epitaxially-grown by offaxis de magnetron sputtering $\mathrm{YBa}_{2} \mathrm{Cu}_{3} \mathrm{O}_{7-\delta}$ (YBCO) films with $J_{c}(H \| c, 77 \mathrm{~K}) \geq 2 \cdot 10^{6} \mathrm{~A} / \mathrm{cm}^{2}$. The mechanism of vortex depinning from growth-induced linear defects, i.e., out-of-plane edge dislocations in low-angle tilt domain boundaries, is shown to describe quantitatively measured $J_{c}(H \| c, T)$. The developed model takes into account a statistical distribution of the dislocation domain boundaries ordered in a network as well as the interdislocation spacing within boundaries. Actual structural features of YBCO film known from HREM data turn out to be extracted from $J_{c}(H \| c, T)$-curves by a fitting procedure within the proposed model.
\end{abstract}

Index Terms-Abrikosov vortices, critical current density, edge dislocations, epitaxial thin films, high-temperature superconductors.

\section{INTRODUCTION}

$\mathbf{E}$ PITAXIALLY-GROWN $c$-axis-oriented single-crystalline $\mathrm{YBa}_{2} \mathrm{Cu}_{3} \mathrm{O}_{7-\delta} \quad(\mathrm{YBCO})$ films exhibit critical current densities, $J_{c}$, two orders of magnitude higher than any kind of bulk samples [1]-[4]. The reason is supposed to be a high density of extended natural linear crystal defects. It is shown that two alternative mechanisms [4] of $J_{c}$-limitation can occur in YBCO films with the highest critical current density, $J_{c}(H \| c, 77 \mathrm{~K}) \geq(2-3) \cdot 10^{6} \mathrm{~A} / \mathrm{cm}^{2}$. They are the depairing/ transparency mechanism and the depinning mechanism. Which one of these mechanisms would be realized, depends on the values of $J_{c}^{\text {transparency }}$ and $J_{c}^{\text {depinning: the lower } J_{c} \text { defines }}$ the acting mechanism [4]. $J_{c}^{\text {transparency }}$ and $J_{c}^{\text {depinning }}$ are in turn determined by the film mosaic domain structure. It is known from HREM data [5] that a multitude of natural linear defects (dislocations) is formed in the single-crystalline epitaxially-grown YBCO films. Out-of-plane edge dislocations, forming low-angle tilt domain boundaries (LAB's) and thus

Manuscript received August 6, 2002. This work was supported in part by the INTAS grant 99-585 and NAS of Ukraine grant VC/95.

V. M. Pan, V. A. Komashko, and C. G. Tretiatchenko are with Institute for Metal Physics, Kiev 03142, Ukraine (e-mail: pan@imp.kiev.ua; ctret@imp. kiev.ua; dixi@carrier.kiev.ua).

E. A. Pashitskii, S. M. Ryabchenko, A. V. Semenov, and Y. V. Fedotov are with Institute of Physics, NAS of Ukraine, Kiev 03028, Ukraine (e-mail: pashitsk@iop.kiev.ua; ryabch@iop.kiev.ua; semenov@iop.kiev.ua).

A. V. Pan and S. X. Dou are with the University of Wollongong, Wollongong, NSW 2522, Australia (e-mail: pan@uow.edu.au).

Digital Object Identifier 10.1109/TASC.2003.812523

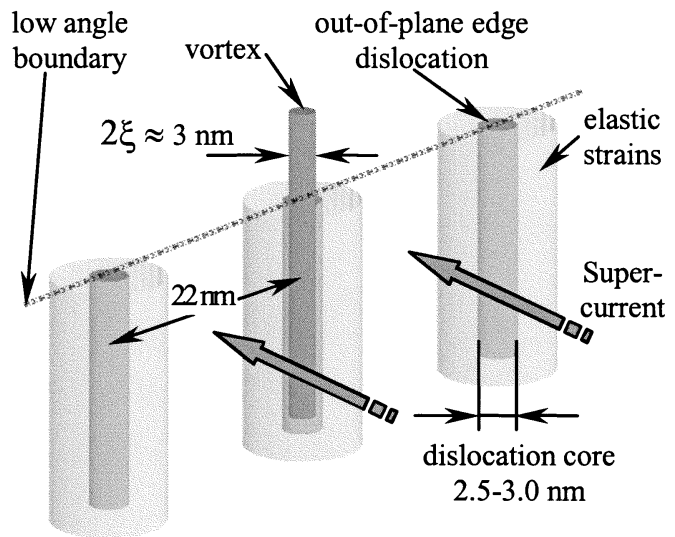

Fig. 1. Out-of-plane edge dislocations serving as pins for Abrikosov vortices in a low-angle $\left(1^{\circ}\right)$ domain boundary with high transparency.

tiny mosaic domains, turned out to be the most important. The domains are usually $20-160 \mathrm{~nm}$ in size and misoriented by about $1^{\circ}$. The mean $2 \mathrm{D}$ in-plane density of such dislocation in the film is $10^{11} \mathrm{~cm}^{-2}$ [5]. LAB's appear to be more or less ordered equidistant rows of parallel dislocations. The dislocations have nonsuperconducting cores surrounded by the stress-strain field area, where $T_{c}$ is locally suppressed. The core diameter is 5-7 interatomic distances in the $a b$-plane, i.e., 2.0-2.5 nm. For the single out-of-plane dislocation at $77 \mathrm{~K}$ the local area with suppressed $T_{c}$ is about $8 \cdot 10^{-13} \mathrm{~cm}^{2}$ [6]. Such rows of normal "holes" in the superconducting matrix form a kind of "fence" network. Normal areas around each of the dislocation lines are potential wells for vortices, when the magnetic field is applied parallel to the LAB's. Dislocation cores provide an extremely strong pinning force resulting in $J_{c}(77 K) \cong(2-3) \cdot 10^{6} \mathrm{~A} / \mathrm{cm}^{2}$. On the other hand, spaces between the dislocations, surrounded by areas with suppressed $T_{c}$, are still transparent for the supercurrent flow across LAB's (Fig. 1). The maximum supercurrent density limited by transparency is the effective depairing current density provided that vortices are pinned strongly enough to prevent any depinning. Variations of linear defects density and of their spatial distribution must result in a particular $J_{c}$-limitation mechanism as well as in $J_{c}(H, T, \theta)$ changes. Transport current measurements of $J_{c}(H)$ angular dependencies show two peaks as the field vector is rotated with respect to current flow direction in the $a b$-plane [7]. Two $J_{c}(H, \theta)$-maxima correspond to $H \| c$ and $H \| a b$, the relative height of the maxima changing with temperature, applied field, electrical field 
criterion $E_{c}$ (i.e., velocity of driven vortex ensemble), as well as with deposition conditions. Out-of-plane edge dislocations are shown to play a crucial role in $J_{c}(H, \mid c, T)$-behavior [6]. Peculiarities of $J_{c}(H \| \mid c)$ are found in the low field range [4]. $J_{c}(H \| c, T)$ is shown to have a plateau $J_{c}=$ const at $H<H_{\min }$, and to be approximated well at $H>H_{\min }$ by the law $J_{c}(H \| c, T) / J_{c}(0, T) \propto \log (H * / H \| c)$, where the characteristic field $H *$ is proportional to reduced temperature $\tau=\left(1-T / T_{c}\right)$. A sharp crossover between plateau and $\log \left(H^{*} / H\right)$ parts in the $J_{c}(H \| c)$-curve [4] is supposed to prove out the existence of transparency-controlled $J_{c}$-limitation in the low-field region. In this paper a model is developed for a consistent quantitative description of the $J_{c}(H \| c, T)$ behavior in YBCO films with $J_{c}>10^{6} \mathrm{~A} / \mathrm{cm}^{2}$. The model should take into account the statistical distribution of the ordered dislocation domain LAB's network as well as the interdislocation spacing within a boundary. The real linear defects structure in YBCO film, known from electron microscopic studies, turns out to be extracted from measured $J_{c}(H \| c, T)$ curves by a proper fitting procedure.

\section{EXPERIMENTAL PROCEDURE}

\section{A. Samples}

YBCO films were deposited onto r-cut optically polished sapphire wafers $51 \mathrm{~mm}$ in diameter. $\mathrm{A} \mathrm{CeO}_{2}$ buffer layer 25-30 $\mathrm{nm}$ thick was deposited by on-axis RF magnetron sputtering. Then the YBCO film was deposited by off-axis dc magnetron sputtering with a use of the rotating substrate holder. The substrate temperature was about $875{ }^{\circ} \mathrm{C}$ and $750{ }^{\circ} \mathrm{C}$ during $\mathrm{CeO}_{2}$ and YBCO deposition, respectively. YBCO film thickness was about $300 \mathrm{~nm}, T_{c}=87 \mathrm{~K}, \Delta T_{c}=0.5 \mathrm{~K}, J_{c}(77 \mathrm{~K})=2.3$. $10^{6} \mathrm{~A} / \mathrm{cm}^{2}$ measured by ac magnetic susceptibility and calculated using the Clem-Sanchez formulae [9]. The structure of the samples was controlled by X-ray diffraction ((00 l)-reflections were checked, the half-width of (005) reflection is $\left.0.22^{\circ}\right)$.

\section{B. Critical Current Measurements}

The magnetization as a function of temperature $T$ and applied magnetic field $H_{a}$ was measured using a Quantum Design MPMS SQUID within the field range of $\left|B_{a}\right| \leq 5 \mathrm{~T}$ and temperature range of $5 \mathrm{~K} \leq T \leq 95 \mathrm{~K}$. The critical current $J_{c}$ was derived from the width of magnetization loops $\Delta M=$ $\left|M^{+}\right|+\left|M^{-}\right|\left(M^{+}\right.$and $M^{-}$are descending and ascending branches, respectively), using the following critical state model formula $J_{c}=2 \Delta M /[w(1-w / 3 l)]$, where $l$ and $w$ are sample length and width, respectively.

The field dependencies of critical currents measured by SQUID at different temperatures from 5 to $80 \mathrm{~K}$ are shown in Fig. 2. It is clearly seen that the plateau regions extend to 0.02-0.10 $\mathrm{T}$ depending on the temperature.

Transport current $J_{c}$-measurements were performed with the thin film $0.25 \mathrm{~mm}$ width bridge manufactured by conventional photolithography and using four-probe technique with the electric field criterion $E_{c}=1 \mu \mathrm{V} / \mathrm{cm}$. The data obtained at $77 \mathrm{~K} \mathrm{co-}$ incide with the SQUID measurements within one order of magnitude.

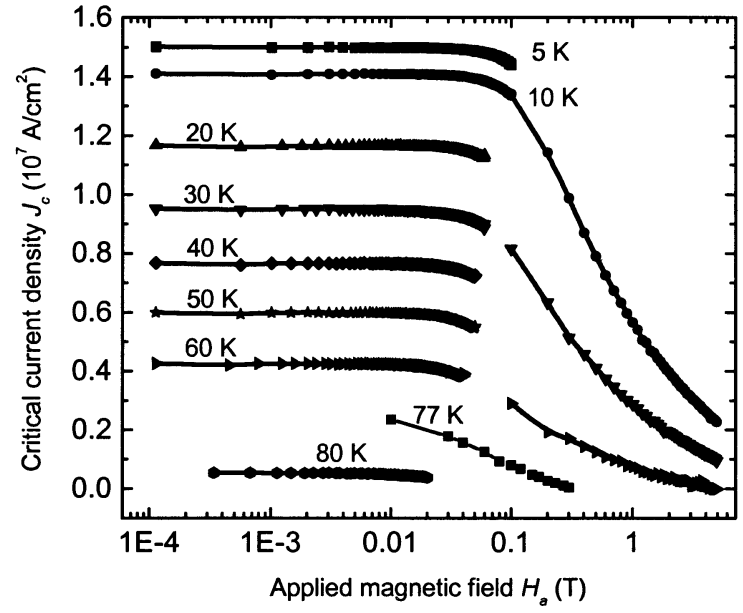

Fig. 2. Critical current density versus applied transverse magnetic field extracted from magnetization curves measured by SQUID magnetometry at different temperatures (except the data for $77 \mathrm{~K}$ measured by transport technique).

\section{Model of $J_{c}(H \| C, T)$ Limitation by Vortex DEPINNING FROM LINEAR PINS}

The model developed in [10] for the $J_{c}$-limitation by vortex depinning from linear pins is based on the following simplifying assumptions: 1) The only effective pins in the film are out-ofplane edge dislocations within LAB's, which form quasiperiodical chains surrounding a multitude of mosaic domains slightly misoriented to each other. 2) Magnetic induction $B=n \phi_{0}$, where $n$ is $2 \mathrm{D}$ density of vortices in the film and $\phi_{0}$ is the flux quantum, coincides with the external magnetic field $H_{\text {ext }}$ applied parallel to the $c$-axis and to the linear pins direction, since the demagnetization factor is practically equal to unity. 3) The following sequence of inequalities holds for the main parameters of the model (Fig. 1):

$$
r_{c} \leq \xi(T)<d \ll(L)>a(H)<\lambda(T)
$$

where $r_{c}$ is the radius of nonsuperconducting dislocation core, $\xi(T)=\xi_{0} \tau^{-(1 / 2)}$ is the coherence length, $d$ is the interdislocation spacing within the $\mathrm{LAB},\langle L\rangle$ is the mean domain cross-size, $a(H) \approx\left(\phi_{0} / H\right)^{1 / 2}$ is the period of undisturbed vortex line lattice (VLL), and $\lambda(T)=\lambda_{0} \tau^{-(1 / 2)}$ is the penetration depth. The critical current in this model is determined from the condition of collective depinning of disturbed VLL from an ensemble of edge dislocations in the LAB's surrounding the domains:

$$
\left(\frac{n_{p}}{n}\right) F_{\text {pind. }}-F_{L i n d .}\left(j_{c}\right)=0
$$

where $n_{p} / n$ is the fraction of vortices pinned at dislocation cores in the absence of current, $F_{L i n d}$. is the Lorentz force acting on an individual vortex

$$
F_{L i n d .}(j)=\frac{\phi_{0} J}{c}
$$


and $F_{p i n d}$ is the maximal individual pinning force. For the case of small insulating cores (see condition (1)) it can be written in the form [11]:

$$
F_{\text {pind }}=\left(\frac{d \varepsilon_{p}(u)}{d u}\right)_{\max }=\frac{9}{32} \sqrt{\frac{2}{3}} \frac{\varepsilon_{0} r_{c}^{2}}{\xi^{3}} \cong 0.23 \frac{\varepsilon_{0} r_{c}^{2}}{\xi^{3}}
$$

where $\epsilon_{0}=\left[\phi_{0} /(4 \pi \lambda)\right]^{2}$. The substitution of (3) and (4) into (2) leads to the expression

$$
\frac{j_{c d e p}(H, \tau)}{j_{c \text { dep }}(0, \tau)=\frac{n_{p}(H)}{n(H)}}
$$

where $j_{c d e p}(0, \tau)=0.23\left(c \phi_{0} r_{c}^{2} /\left(4 \pi \lambda_{0}\right)^{2} \xi_{0}^{3}\right) \tau^{5 / 2}$.

To obtain the accommodation function (5) one must take into account the distortion effect of the linear pin ensemble on the equilibrium VLL. A vortex is assumed to be pinned, if the pinning energy $\left|\epsilon_{\text {pin }}\right|$ exceeds the elastic energy increase due to $2 \mathrm{D}$ shear displacement of the vortex from its regular position in the VLL site to the nearest pinning center. So, the critical distance $\delta$ for a vortex to be pinned can be found from the condition $\left|\epsilon_{\mathrm{pin}}\right| \geq C_{66} \delta^{2}$, where $C_{66}$ is the elastic shear modulus of VLL [12]. Assuming a core-pinning on dislocations with small insulating cores one can find: $\delta=\left(2 r_{c} / \xi_{0}\right)\left(\phi_{0} \tau / H\right)^{1 / 2}$.

We assume further that the film area is completely filled with rectangular domains with an independent random distribution of dimensions $L_{x}$ and $L_{y}$, which is described by the product of probability density functions $P\left(L_{x}\right)$ and $P\left(L_{y}\right)$

The probability density for a site of regular VLL to fall within a domain with dimensions $L_{x}$ and $L_{y}$ is $W\left(L_{x}, L_{y}\right) \propto$ $L_{x} L_{y} P\left(L_{x}\right) P\left(L_{y}\right)$. The probability that this site will be closer than a distance $\delta$ to the LAB would be equal to the ratio of the area of a strip of $\delta$ width near the boundaries of the grain to the area $L_{x} L_{y}$ of the whole grain:

$\tilde{P}\left(L_{x}, L_{y}, \delta\right)= \begin{cases}\frac{L_{x} L_{y}-\left(L_{x}-2 \delta\right)\left(L_{y}-2 \delta\right)}{L_{x} L_{y}} & \\ 1, & \min \left(L_{x}, L_{y}\right) \leq 2 \delta .\end{cases}$

The model can be improved for the higher field region explicitly taking into account LAB's discreteness (distance $d$ between dislocations). This will reduce the trapping area for vortices by a geometrical factor $f(d / 2 \delta)$ :

$$
\begin{aligned}
& f(x)=\frac{1}{2}\left(\sqrt{1-x^{2}}+\frac{\arcsin (x)}{x}\right) \text { for } x \leq 1, \\
& f(x)=\frac{\pi}{4 x} \text { for } x \geq 1 .
\end{aligned}
$$

Finally, we obtain the following expression for the fraction of pinned vortices:

$$
\frac{n_{p}}{n}=\int_{0}^{\infty} d L_{x} \int_{0}^{\infty} d L_{y} W\left(L_{x}, L_{y}\right) \tilde{P}\left(L_{x}, L_{y}, \delta\right) .
$$

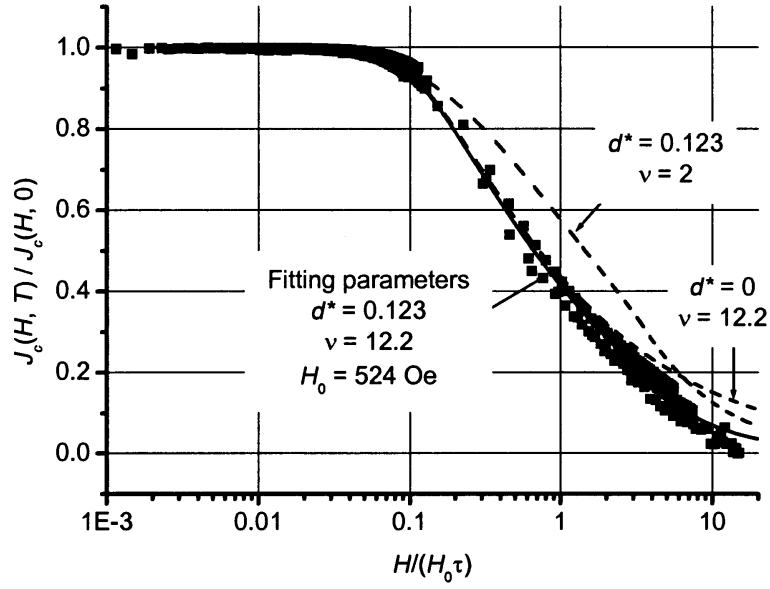

Fig. 3. Reduced field dependencies of the critical current. Solid line shows the fit of experimental data for 10,30 and $60 \mathrm{~K}$. Dashed lines are calculated dependencies for the wider size distribution $(\nu=2)$ and for continuous LAB's $(d=0) . d *$ is dimensionless dislocation spacing $d *=d /\langle L\rangle$.

In particular, for the statistical $\Gamma$-distribution [4]

$$
P(L)=\frac{\mu^{\nu}}{\Gamma(\nu)} L^{\nu-1} e^{-\mu L}
$$

the following expression is obtained:

$$
\begin{aligned}
\frac{n_{p}}{n}= & -\left[1-f\left(\frac{d}{2 \delta}\right)\right]\left[\frac{\Gamma(\nu, 2 \mu \delta)}{\Gamma(\nu)}\right]^{2} \\
& -f\left(\frac{d}{2 \delta}\right)\left[\frac{\Gamma(\nu, 2 \mu \delta)-2 \mu \delta \Gamma(\nu-1,2 \mu \delta)}{\Gamma(\nu)}\right]^{2}
\end{aligned}
$$

where $\mu=\langle L\rangle / \sigma^{2}, \nu=\mu\langle L\rangle, \sigma$ is the width (dispersion) of the distribution function, $\Gamma(\nu)$ and $\Gamma(\alpha, \beta)$ are complete and noncomplete gamma-functions.

\section{DiscusSION}

As it follows from the model, the dependencies of the critical current normalized by the plateau value depend on the magnetic field and temperature only through the parameter of vortex trapping by dislocations $\delta \propto(\tau / H)^{1 / 2}$. This means that $J_{c}(H \| c, T) / J_{c}(0, T)$-dependencies upon some function of $\delta$ must be invariant. In particular, log-linear plots of normalized $J_{c}$ versus $H /\left(H_{0} \tau\right)$ for different fixed temperatures must coincide. It is seen (Fig. 3) that this is the case with good precision. $H_{0}$ is introduced for convenience from the condition $2 \mu \delta \equiv \nu(2 \delta /\langle L\rangle) \equiv \nu\left(H_{0} \tau / H\right)^{1 / 2}$. Taking into account the definition of $\delta$, one can find $H_{0}=8 r_{c}^{2} \phi_{0} /\left(\xi_{0}^{2}\langle L\rangle^{2}\right)$. The experimental data have been fitted by (10) with $H_{0}, \nu$ and $d /\langle L\rangle$ chosen as independent fitting parameters. For the maximal temperature of $60 \mathrm{~K}$ and highest fields a slight deflection from the universal fit can be due to an influence of thermal flux creep [11], which was not taken into account. The creep contribution increases with temperature and decreases with critical current.

The parameter $\nu=12.2$ found from the fit corresponds to a rather narrow distribution of domain size (Fig. 4). The parameter $\alpha=\partial\left[J_{c}(H) / J_{c}(0)\right] / \partial \ln \left(H_{0} \tau / H\right)$ taken in the inflection point [10] was estimated to be 0.25 , that is higher for the investigated film than it was in $[10](\alpha \approx 0.18-0.24)$. The mean domain size can be estimated from the value of $H_{0}=524 \mathrm{Oe}$. As- 


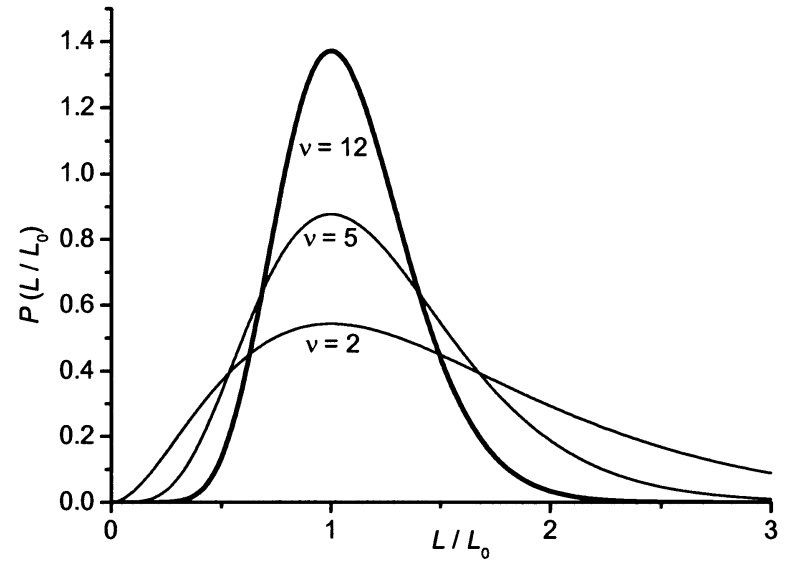

Fig. 4. Model domain size distributions. The value $L_{0}$ corresponds to peak positions. Areas under the curves are normalized to be unity. The present experimental data are best fitted with $\nu=12.2$. The data [10] correspond to $\nu \approx 2$.

suming $r_{c}=0.5 \xi_{0},\langle L\rangle$ is estimated to be $280 \mathrm{~nm}$ and through the third fitting parameter $d \approx 35 \mathrm{~nm}$. Using Frank's relationship, the estimated misorientation angle $\theta \approx 0.65^{\circ}$. This extremely low value explains the absence of visual crossover to the LAB transparency mechanism of $J_{c}$-limitation [4] at low magnetic fields observed for the pulse-laser deposited film investigated earlier [10].

\section{CONCLUSION}

$J_{c}(H \| c, T)$ were measured by SQUID-magnetometry, ac magnetic susceptibility, and dc transport current techniques in the single-crystalline epitaxially-grown YBCO films with $J_{c}(77 K) \geq 2 \cdot 10^{6} \mathrm{~A} / \mathrm{cm}^{2}$. Vortex depinning from growth-induced linear defects, i.e., out-of-plane edge dislocations in low-angle tilt domain boundaries, is shown to describe the quantitatively measured $J_{c}(H \| c, T)$. The developed model taking into account a statistical distribution of the dislocation domain boundaries ordered in a network, as well as the interdislocation spacing within boundaries, allows us to obtain the domain structure parameters by a fitting procedure.

\section{REFERENCES}

[1] V. M. Pan, V. S. Flis, V. A. Komashko, O. P. Karasevska, V. L. Svetchnikov, M. Lorenz, A. N. Ivanyuta, G. A. Melkov, E. A. Pashitskii, and H. W. Zandbergen, "Linear defects in epitaxial $\mathrm{Y}-\mathrm{Ba}-\mathrm{Cu}-\mathrm{O}$ films: Their role in anisotropic vortex pinning and microwave surface resistance," IEEE Trans. Appl. Supercond., vol. 11, pp. 3960-3963, 2001.

[2] B. Dam, J. M. Huijbregste, F. C. Klaassen, R. C. F. Van der Geest, G. Doornbos, J. H. Rector, A. M. Testa, S. Freisem, J. C. Martinez, B. Stäuble-Pümpin, and R. Griessen, "Origin of high critical currents in $\mathrm{YBa}_{2} \mathrm{Cu}_{3} \mathrm{O}_{7-\delta}$ superconducting thin films," Nature, vol. 399, pp. 439-442, 1999.

[3] E. Mezzetti, R. Gerbaldo, G. Ghigo, L. Gozzelino, B. Minetti, C. Camerlingo, A. Monaco, G. Cuttone, and A. Rovelli, "Control of the critical current density in $\mathrm{YBa}_{2} \mathrm{Cu}_{3} \mathrm{O}_{7-d}$ films by means of intragrain and correlated defects," Phys. Rev. B, vol. 60, pp. 7623-7630, 1999.

[4] E. A. Pashitskii, V. I. Vakaryuk, S. M. Ryabchenko, and Yu. V. Fedotov, "Temperature dependence of the critical current in high $-T_{c}$ superconductors with low-angle boundaries between crystalline blocks," Low Temp. Phys., vol. 27, pp. 96-102, 2001.

[5] V. L. Svetchnikov, V. M. Pan, Ch. Traeholt, and H. Zandbergen, "Formation of edge dislocations in thin epitaxial YBCO films," IEEE Trans. Appl. Supercond., vol. 7, pp. 1396-1398, 1997.

[6] V. M. Pan, V. S. Flis, O. P. Karasevska, V. I. Matsui, I. I. Peshko, V. L. Svetchnikov, M. Lorenz, A. N. Ivanyuta, G. A. Melkov, E. A. Pashitskii, and H. W. Zandbergen, "Effect of growth-induced linear defects on high frequency properties of pulse-laser deposited $\mathrm{YBa}_{2} \mathrm{Cu}_{3} \mathrm{O}_{7-\delta}$ Films," $J$. Superconductivity: Inc. Novel Magn., vol. 14, pp. 109-118, 2001.

[7] W. Schindler, B. Roas, G. S. Ischenko, L. Schultz, and H. Gerstenberg, "Anisotropic enhancement of the critical current density of epitaxial $\mathrm{YBa}_{2} \mathrm{Cu}_{3} \mathrm{O}_{7-x}$ films by fast neutron irradiation," Physica C, vol. 169, pp. $117-122,1990$.

[8] V. M. Pan, A. L. Kasatkin, V. L. Svetchnikov, V. A. Komashko, A. G. Popov, A. Yu. Galkin, H. C. Freyhardt, and H. W. Zandbergen, "Critical current density in highly biaxially-oriented ybco films: can we control $J_{c}(77 \mathrm{~K})$ and optimize up to more than $10^{6} \mathrm{~A} / \mathrm{cm}^{2}$," IEEE Trans. Appl. Supercond., vol. 9, pp. 1535-1538, 1999.

[9] J. R. Clem and A. Sanchez, "Hysteretic AC losses susceptibility of thin superconducting disks," Phys. Rev. B, vol. 50, pp. 9355-9362, 1994.

[10] Y. V. Fedotov, S. M. Ryabchenko, E. A. Pashitskii, A. V. Semenov, V. I. Vakaryuk, V. M. Pan, and V. S. Flis, "Magnetic-field and temperature dependence of the critical current in thin epitaxial films of the hightemperature superconductor $\mathrm{YBa}_{2} \mathrm{Cu}_{3} \mathrm{O}_{7-\delta}$," Low Temp. Phys., vol. 28, pp. 172-175, 2002.

[11] E. A. Pashitskii and V. I. Vakaryuk, "Pinning of Abrikosov vortices on dislocations and the critical current in high-temperature superconductors," Low Temp. Phys., vol. 28, pp. 96-99-96-99, 2002.

[12] G. Blatter, M. V. Feigel'man, V. B. Geshkenbein, A. I. Larkin, and V. M. Vinokur, "Vortices in high-temperature superconductors," Rev. Mod. Phys., vol. 66, pp. 1125-1139, 1994. 